\title{
Ampliando Elementos da Educação Médica: Morbidade Referida em Universitários de Medicina
}

\author{
Widening Elements of Medical Education: \\ Refered Morbidity in Medicine Freshmen
}

PALAVRAS-CHAVE

- Morbidade;

- Estudantes de Medicina;

- Medicamentos;

- Educação Médica.

KEY-WORDS

- Morbidity;

- Students, Medical;

- Medicaments;

- Education, medical.

Recebido em: 01/06/2004

Reencaminhado em: 17/11/2005

Aprovado em: 13/01/2006

$15 \mid \frac{\text { REVISTA BRASILEIRA DE EDUCAÇÃO MÉDICA }}{\text { Rio de Janeiro, v.30, no } 1 \text {, jan./abr. } 2006}$
Marcelo Conte ${ }^{1}$ Aguinaldo Gonçalves ${ }^{2}$

\section{RESUMO}

Este estudo teve por objetivo identificar e explorar a morbidade referida de um grupo de calouros de Medicina. Trata-se de estudo transversal seriado, que considera o sexo como variável independente e a morbidade referida como dependente. Foram investigados 288 calouros de Medicina da Unicamp durante três anos consecutivos, tendo-se coletado os dados por meio de anamnese e semiologia clínica. No plano analítico, as comparações entre proporções foram processadas pela estatística qui-quadrado, e interpretadas ao nível de 5\% de significância. Os principais resultados revelaram diferença significativa somente em relação ao uso atual de medicamentos, apontando que o sexo feminino referiu consumo maior de remédios do que o masculino. De maneira geral, estes dados diferem dos disponíveis na literatura, apontando, sobretudo, a especificidade da população estudada.

\begin{abstract}
This study aimed to identify and explore referred morbidity in a group of medicine freshmen. It is a serial transversal study, considering sex as independent variable and referred morbidity as a dependent one. Two hundred eighty eight medicine freshmen from Unicamp-Brazil have been investigated during three years. Data were collected by anamnesis and clinical semiology. Statistical analysis included comparisons of proportions according to the $\chi^{2}$ test at a significance level of 5\%. Main results showed significant differences only on medicament consumption, with females consuming more than males.
\end{abstract}

\footnotetext{
${ }^{1}$ Coordenador do Núcleo de Estudos e Pesquisas em Ciências do Esporte; Curso de Educação Física do Uirapuru Superior, Escola Superior de Educação Física de Jundiaí, Jundiaí, São Paulo, Brasil.

${ }^{2}$ Coordenador do Grupo de Saúde Coletiva/Epidemiologia e Atividade Física da Faculdade de Educação Física, Universidade Estadual de Campinas, Campinas, São Paulo, Brasil.
} 


\section{INTRODUÇÃO}

No contexto da Educação Médica, o universo de agravos à saúde que atinge a população de estudantes, ou seja, a morbidade, é um importante aspecto a ser considerado ${ }^{1}$. Ele se relaciona, basicamente, a dois indicadores epidemiológicos: i) prevalência, correspondente ao número total de casos existentes de determinado agravo em certo período e local; ii) incidência, referente ao número de casos novos de certa doença em local e período específicos ${ }^{2}$. De fato, observa-se que as doenças encontradas na sociedade contemporânea representam um quadro tão complexo quanto as próprias características da sociedade. Desta forma, as doenças formam um espectro diversificado: infecciosas e crônico-degenerativas, transmissíveis ou não transmissíveis, curáveis ou incuráveis, preveníveis ou não, próprias da infância ou da velhice, freqüentes nos países desenvolvidos ou típicas do Terceiro Mundo $^{3}$. Em termos da nossa realidade, o panorama da Saúde Coletiva brasileira mostra-se compatível com a tendência dos padrões mundiais ${ }^{4}$, no sentido de que os grupos de doenças revelam a pluralidade de situações a que a humanidade está submetida nesta transição de milênio.

Em relação aos jovens brasileiros, as mortes provocadas por acidentes e violência, fatores também denominados causas externas, provocam cerca de $65 \%$ dos óbitos, ocorridos entre os 10 e 29 anos $^{3}$. No que se refere aos acidentes de trânsito (AT), responsáveis por elevado índice de incapacidade e uso intensivo de serviços médicos de alto custo, verificase a predominância de acidentados do sexo masculino na faixa entre 20-29 anos 5 .

Entre alunos do $5^{\circ}$ e $6^{\circ}$ anos de Faculdade de Medicina, observou-se, em Ribeirão Preto (SP), o desrespeito esporádico a semáforos, a condução de veículos sob o efeito de álcool e a participação em "rachas" como as infrações de trânsito mais freqüentes neste segmento, considerando o sexo masculino significativamente associado $^{6}$. Relata-se ainda que 20,5\% dos estudantes estiveram envolvidos em AT, sendo que em 4,1\% deles ocorreu intervenção hospitalar em algum dos passageiros.

Evidentemente, tais atitudes não são exclusivas de universitários brasileiros. Uma investigação francesa com grupos de estudantes atletas revelou que os estudantes ativos apresentavam comportamento de risco à saúde significativamente maior do que os inativos, em relação aos seguintes aspectos: i) consumo médio de álcool; ii) condução de automóveis sob influência de álcool ou outras drogas; iii) direção automotiva sem habilitação; iv) não utilização de cinto de segurança no carro, ou capacete em moto; v) ausência do uso de contraceptivos; vi) freqüência de doenças sexualmente transmissíveis; vii) número maior de parceiros sexuais ${ }^{7}$.
Outro sério problema de saúde pública entre nossos jovens é a Síndrome da Imunodeficiência Adquirida (aids) - (o Brasil situa-se entre os quatro primeiros países do mundo com o maior número de casos registrados): desde o início da epidemia, o grupo mais atingido tem sido o de 20 a 39 anos. Ao longo do tempo, observa-se progressivo aumento da freqüência relativa de pacientes mais jovens (20-29), com tendência a estabilização a partir de $1991^{8}$.

A infecção pelo HIV durante a adolescência é importante e proporcionalmente maior entre o sexo feminino. A maioria das moças contaminadas da cidade do Rio de Janeiro (RJ) tem baixo grau de escolaridade, e a principal forma de contaminação é o contato heterossexual; em decorrência, o estudo sugere a necessidade de direcionar as campanhas de prevenção e controle a esses grupos específicos ${ }^{9}$. Neste sentido, informação e escolaridade parecem insuficientes para a adoção de medidas preventivas, pois $40,4 \%$ dos estudantes de Medicina da Universidade de Pernambuco, "apesar de terem conhecimentos sobre as formas de prevenção da aids, nunca fizeram uso de preservativos em suas relações sexuais"10.

O panorama dos agravos mais freqüentes nos jovens revela outras dimensões, a partir da metodologia empregada na investigação. Em nosso meio, um estudo de morbidade referida entre calouros de curso de Medicina ${ }^{11}$, em que se utilizaram anamnese e semiologia clínica, verificou: maior freqüência relativa de doenças e internações hospitalares dos rapazes comparativamente às moças e predominância de agravos respiratórios e osteomusculares em ambos os sexos. Os agravos mencionados na investigação dos estudantes de Medicina da Unicamp são diferentes daqueles que afligem grande parte da população jovem brasileira, parte em virtude da especificidade desse segmento populacional e parte pelo tipo de metodologia adotada na identificação e registro dessa morbidade.

Assim, a juventude pode ser considerada um dos segmentos especialmente sobrecarregados e/ou desprotegidos em nosso sistema social e prioritário na demanda de esforços para proteção e promoção da saúde ${ }^{12}$. Tendo em conta tais evidências, o objetivo da presente investigação é identificar e explorar a morbidade referida entre calouros de Medicina da Unicamp.

\section{MÉTODO}

O estudo se tipifica como transversal seriado ${ }^{1}$, em termos de detectar simultaneamente as variáveis de interesse, em três anos consecutivos. Como variável independente, identificou-se o sexo, e como dependente, a morbidade referida dos calouros. 
Tomou-se como população de estudo o conjunto dos calouros do curso de Medicina matriculados na Universidade Estadual de Campinas (Unicamp), um total de 288 pessoas freqüentes à Disciplina de Educação Física Desportiva, com a respectiva distribuição da média e desvio-padrão da idade, segundo sexo, apresentada na Tabela 1.

A coleta de dados foi efetuada em três anos consecutivos, ou seja, compreendeu informações referentes a três turmas sucessivas de calouros, nos primeiros semestres letivos dos anos de 1997, 1998 e 1999.

Os registros sobre morbidade referida foram obtidos por meio de anamnese e semiologia clínica de rastreamento, empregadas dirigidamente pela respectiva área técnica especializada da Universidade (Cecom - Centro de Saúde da Comunidade), segundo suas condutas profissionais habituais. Importa registrar que os dados obtidos fazem parte dos procedimentos habituais dirigidos aos alunos em todos os cursos dessa natureza ministrados pelo Grupo de Saúde Coletiva/Epidemiologia e Atividade Física da Unicamp, diferindo das turmas de outros cursos apenas segundo as técnicas analíticas empregadas. Ainda assim, tomou-se o cuidado de informar aos alunos que os dados obtidos na pesquisa seriam objeto de estudo. Também foi solicitado que cada participante assinasse o termo de Consentimento Livre Esclarecido Informado, observando adoção integral das recomendações da Declaração de Helsink ${ }^{13}$.

Como procedimento analítico, visando comparações entre proporções, foi utilizada a estatística qui-quadrado, segundo programa informatizado corrente e interpretadas ao nível de 5\% de significância. Tais resultados foram expressos sob a forma de apresentação tabular de acordo com o preconizado pelas normas vigentes ${ }^{14}$.

\section{RESULTADOS}

A Tabela 1 apresenta a distribuição da média e desviopadrão da idade dos alunos estudados, segundo sexo. A Tabela 2 mostra a distribuição das referências investigadas, apontando diferença estatística somente em relação ao uso atual de medicamentos, que é maior no sexo feminino.

TABELA 1

Distribuição das medidas descritivas da idade (em anos) dos alunos estudados, segundo sexo

\begin{tabular}{l|c|c|c}
\hline \multirow{2}{*}{ Medidas Descritivas } & \multicolumn{2}{|c}{ Sexo } & \multirow{2}{*}{ Total } \\
\cline { 2 - 4 } & Masculino & Feminino & 288 \\
\hline $\mathrm{N}$ & 144 & 144 & 18 \\
\hline Mediana & 18 & 18 & 18,52 \\
\hline Média & 18,54 & 18,46 & 01,13 \\
\hline Desvio-Padrão & 01,21 & 01,00 & 17 \\
\hline Limite Inferior & 17 & 17 & 23 \\
\hline Limite Superior & 23 & 22 & \\
\hline
\end{tabular}

\section{DISCUSSÃO}

Os dados decorrentes da morbidade referida apresentaram diferença estatística em relação ao uso atual de medicamentos. Observou-se que as garotas mencionam maior consumo de remédios do que os rapazes, sobretudo devido à elevada utilização de drogas que atuam no sistema endócrino, nutricional e metabólico. De fato, neste conjunto situa-se o segundo grupo mais importante na morbi-mortalidade dos jovens do Rio de Janeiro e São Paulo a partir de 199115, porém às custas de acometimentos no sexo masculino, especificamente devido aos casos de aids, que, segundo as notificações, provêm de homens em cerca de $80 \%$ do total de $\operatorname{casos}^{16}$. Na tentativa de entender essa situação, é de cogitar que, no grupo dos estudantes, o maior consumo de medicamentos no sexo feminino se explique pelo uso de anticoncepcionais, diferentemente da população em geral, mais exposta a outros tipos de agravos.

No entanto, um estudo desenvolvido na Suécia apontou como preocupante o uso abusivo de antidepressivos entre mulheres, os quais estavam associados a muitos casos de overdose ${ }^{17}$. Além disso, um levantamento realizado com recémingressos em instituição de ensino superior do interior paulis- 
TABELA 2

Distribuição dos alunos estudados, por referências investigadas, segundo sexo

\begin{tabular}{|c|c|c|c|c|c|c|c|c|}
\hline \multirow{3}{*}{$\begin{array}{l}\text { Referência } \\
\text { investigada }\end{array}$} & & \multicolumn{4}{|c|}{ Sexo } & \multirow{2}{*}{\multicolumn{2}{|c|}{ Total }} & \multirow{3}{*}{$\chi^{2}$} \\
\hline & & \multicolumn{2}{|c|}{ Masculino } & \multicolumn{2}{|c|}{ Feminino } & & & \\
\hline & & $\mathbf{N}$ & $\%$ & $\mathbf{N}$ & $\%$ & $\mathbf{N}$ & $\%$ & \\
\hline \multirow{3}{*}{ Morbidade } & não & 115 & 39,93 & 105 & 36,45 & 220 & 76,38 & \multirow{3}{*}{$\chi_{(1 ; 0,05)}^{2}=1,92 \mathrm{n} . \mathrm{s}$} \\
\hline & $\operatorname{sim}$ & 29 & 10,07 & 39 & 13,55 & 68 & 23,62 & \\
\hline & total & 144 & 50,00 & 144 & 50,00 & 288 & 100,00 & \\
\hline \multirow{3}{*}{$\begin{array}{l}\text { Morbidade } \\
\text { Hospitalar }\end{array}$} & não & 103 & 3576 & 108 & 37,50 & 211 & 73,26 & \multirow{3}{*}{$\chi_{(1 ; 0,05)}^{2}=0,44 \mathrm{n} \cdot \mathrm{s}$} \\
\hline & $\operatorname{sim}$ & 41 & 1424 & 36 & 12,50 & 77 & 26,74 & \\
\hline & total & 144 & 50,00 & 144 & 50,00 & 288 & 100,00 & \\
\hline \multirow{3}{*}{$\begin{array}{l}\text { Uso Atual de } \\
\text { Medicamentos }\end{array}$} & não & 128 & 44,45 & 115 & 39,93 & 243 & 84,38 & \multirow{3}{*}{$\chi_{(1 ; 0,05)}^{2}=4,45^{*}$} \\
\hline & $\operatorname{sim}$ & 16 & 5,55 & 29 & 10,07 & 45 & 15,62 & \\
\hline & total & 144 & 50,00 & 144 & 50,00 & 288 & 100,00 & \\
\hline
\end{tabular}

* = significativo; n.s. = não significativo.

ta constatou que, em ambos os sexos, somente $33 \%$ dos discentes relataram presença de dor; o consumo maior de medicamentos foi do sexo feminino, sendo referidos como os mais utilizados a Aspirina e o Buscopan ${ }^{18}$. Por outro lado, uma investigação realizada, na década de 1980, com alunos da área Biomédica da Universidade de São Paulo sinalizava a crescente utilização de energizantes e a redução do uso de psicoestimulantes ou anorexígenos e dos ansiolíticos naquela população ${ }^{19}$.

No presente levantamento, não foi encontrada diferença entre a morbidade segundo sexo. Contudo, em estudantes de Odontologia de Araraquara ${ }^{20}$, verificou-se prevalência de hipertensão de $4,3 \%$, com predomínio no sexo masculino, não obesos, na faixa de 20 a 28 anos, cursando o primeiro ano.

Destaca-se também a especificidade da população estudada. Em primeiro lugar, pela própria idade, questão já apontada neste texto. Por outro lado, é notório que alunos de curso de Medicina apresentam aspectos peculiares quando comparados com os demais - sobretudo, carência de disponibilidade de tempo livre para lazer ${ }^{21}$. De fato, um estudo epidemiológico realizado em curso de Medicina de uma universidade peruana (alunos de $4^{\circ}, 5^{\circ}$ e $6^{\circ}$ anos) revelou que a prevalência de transtornos mentais $(45,6 \%)$ é maior do que a encontrada na população geral, destacando a depressão $(31,6 \%)$ e a ansiedade $(22,4 \%)^{22}$.

Deve-se também considerar que, devido à privilegiada situação de que desfrutam, os universitários serão lideranças naturais em suas inserções profissionais futuras ${ }^{23}$ e poderão se tornar formadores de opinião pública em saúde. Outro aspecto relevante e particular são as características do processo de seleção e do curso, que expõem os calouros a estresse diferenciado, devido à competitividade do vestibular ${ }^{24}$.

Neste panorama, considera-se o aluno de escolas médicas em posição de maior risco e suscetibilidade ao uso e abuso de drogas, devido ao maior acesso a elas ${ }^{25}$. Contudo, segundo levantamentos nacionais, o uso de maconha, cocaína, tranqüilizantes, anfetaminas e solventes entre estudantes de $\mathrm{Me}$ dicina é menor do que em outros universitários, embora o consumo de álcool seja significantemente mais elevado ${ }^{26}$.

\section{REFERÊNCIAS}

1. Pereira MG. Epidemiologia: teoria e prática. Rio de Janeiro: Guanabara Koogan; 1995.

2. Gonçalves A, Gonçalves NNS. Saúde e doença: conceitos básicos. Revista Brasileira de Ciência e Movimento. 1988; 2(2):48-56.

3. Barros MBA. Doenças da vida moderna. In: Kupstas M et al. Saúde em debate. São Paulo: Moderna; 1997. p.135-148.

4. Gonçalves A et al. Saúde Coletiva/Atividade Física: uma relação controversa. In: Gonçalves A et al. Saúde Coletiva e Urgência em Educação Física. Campinas: Papirus, 1997. p.153-158. 
5. Soares, DFPP. Características das vítimas de acidentes de trânsito com motocicleta em área urbana. Procedente do $4^{\circ}$ Congresso Brasileiro de Epidemiologia; 1998, ago 23-29: Rio de Janeiro, Brasil.

6. Jorge LL et al. Estudo sobre o comportamento em relação ao trânsito entre estudantes de medicina. Procedente do $4^{\circ}$ Congresso Brasileiro de Epidemiologia; 1998, ago 23-29: Rio de Janeiro, Brasil.

7. Butler J. Sport et santé: pour le meilleur et sans le pire. Cinésiologie. 1992; 31(145): 238-42.

8. Castilho EA, Cheque P. A epidemia de Aids no Brasil. Procedente do Simpósio Satélite: A epidemia de Aids no Brasil: situação e tendências/Coordenação Nacional de DST e Aids; 1997 mar 15-20: Brasília (DF): Ministério da Saúde; 1997.

9. Sanches K. Perfil epidemiológico dos casos de Aids entre adolescentes no Estado do Rio de Janeiro. Procedente do $4^{\circ}$ Congresso Brasileiro de Epidemiologia; 1998, ago 23-29: Rio de Janeiro, Brasil.

10. Brito AM et al. Sexualidade e Aids: a percepeção dos estudantes de medicina da Universidade de Pernambuco. Procedente do $4^{\circ}$ Congresso Brasileiro de Epidemiologia; 1998, Ago 23-29: Rio de Janeiro-RJ, Brasil.

11. Gonçalves A et al. A saúde da geração saúde: pesquisa e ensino sobre capacidades físicas e referências a hábitos e morbidade dos calouros da Faculdade de Ciências Médicas da Unicamp. Revista Brasileira de Atividade Física e Saúde. 1997; 2(4):41-58.

12. Breilh JA. Epidemiologia na humanização da vida: convergências e desencontros das correntes. In. Barradas MR et al. Equidade e saúde: contribuições da epidemiologia. Rio de Janeiro: FIOCRUZ/ABRASCO; 1997.

13. Brasil. Ministério da Saúde. Fundação Nacional da Saúde. Informe Epidemiológico do SUS. 1996; 5(2): s3.

14. Padovani CR. Estatística na metodologia da investigação científica. Botucatu (SP): Instituto de Biociências; 1995.

15. Vermelho LL, Jorge MHPM. Mortalidade de jovens: análise do período de 1930 a 1991 (a transição epidemiológica para a violência). Rev Saúde Pública. 1996; 30(4): 319-31.

16. Façanha MC et al. AIDS em mulheres em fortaleza-CE, 1987-1997. Procedente do 4 ${ }^{\circ}$ Congresso Brasileiro de Epidemiologia; 1998, ago 23-29: Rio de Janeiro, Brasil.

17. Bäckman J et al. Use of antidepressants in deliberate selfpoisoning: psychiatric diagnoses and drugs used between 1987 and 1997 in Lund, Sweden. Soc Psychiatry Psychiatr Epidemiol. 2003; 38 (12):684-689.
18. Silva Netto CR. Consumo de medicamentos por Universitários. Revista da Faculdade de Odontologia de Lins. 1991;4(1):18-22.

19. Delucia R, Planeta CS. Padrões de uso de psicoestimulantes e energizantes por universitários: análise do período 19831988. Rev ABP-APAL. 1989;11(3):111-113.

20. Loffredo LCM. Prevalência de hipertensäo arterial sistêmica em estudantes da Faculdade de Odontologia de Araraquara - UNESP. Rev Odontol. UNESP; 2003; 32(2):99104.

21. Nunes ED. Sobre a sociologia da saúde: origens e desenvolvimento. São Paulo: Hucitec: 1999.

22. Cambiazo G. Aplicación del MINI como orientación diagnóstica psiquiátrica en estudiantes de medicina de la Universidad Peruana Cayetano Heredia: informe preliminar epidemiológico. Rev Med Hered. 2002; 13(1):19-25.

23. Pires GL. Perspectivas político-pedagógicas para a Educação Física no $3^{\circ}$ Grau. Procedente do 5ำ Seminário Nacional de Educação no 3ํㅡㅁ Grau, 1996 nov 10-15: Florianópolis, Brasil.

24. Gonçalves A et al. Educação Física Curricular para o Curso de Medicina: construindo autonomia em Saúde Coletiva e Atividade Física através de ensino e pesquisa. Procedente do $6^{\circ}$ Congresso Brasileiro de Saúde Coletiva; 2000 ago 2830, Salvador, Brasil.

25. Schwartz RH et al. Cocaine and marijuana use by medical students before and during medical school. Arch Intern Med. 1990; (150): 883-6.

26. Andrade AG et al. Fatores de risco associados ao uso de álcool e drogas na vida, entre estudantes de medicina do Estado de São Paulo. Revista ABP-APAL 1997; 19(4): 117-26.

\section{Endereço para correspondência}

Marcelo Conte

Núcleo de Estudos e Pesquisas em Ciências do Esporte Curso de Educação Física/Uirapuru Superior Av. Bento Mascarenhas Jequitinhonha, 633 - Jardim Panorama - 18030-330 - Sorocaba - SP e-mail: conte@uirapuru.edu.br 